\title{
Competencia digital del profesorado. Estrategias de evaluación en tiempos líquidos
}

Teachers' digital competence. Assessment strategies in liquid times

\author{
Antonio Palacios-Rodríguez ${ }^{1}$ \\ Sandra Martínez-Pérez ${ }^{2}$ \\ Universidad de Sevilla \\ Bárbara Fernández-Robles ${ }^{3}$ \\ Universidad Isabel I
}

Recibido: 10.08 .2021

Aceptado: 20.10.2021

\section{Resumen}

La evaluación es una pieza fundamental en los procesos formativos de enseñanza-aprendizaje. Profesorado y estudiantado están llamados a reflexionar y valorar en torno al despliegue de estrategias de evaluación en entornos líquidos. El objetivo de este trabajo es identificar herramientas digitales en los procesos de enseñanza-aprendizaje como recursos para la adquisición de competencias y para evaluación educativa. Para ello, en primer lugar, se aborda el concepto de evaluación, seguido de las técnicas y estrategias que se utilizan en los procesos formativos. Para continuar con la identificación de herramientas digitales y su puesta en

\footnotetext{
1 aprodriguez@us.es https://orcid.org/0000-0002-0689-6317

2 smartinezperez@us.es

https://orcid.org/0000-0002-7458-1077

${ }^{3}$ bfernandezrobles@gmail.com https://orcid.org/0000-0002-1760-392X

Volumen 2. Número 2. Julio - Diciembre 2022 ISSN: 2745-0341 (En línea)
} 
marcha los procesos de enseñanza - aprendizaje, identificando aquellas que, como dicentes, desplegamos en nuestras prácticas educativas. Finalmente señalamos, a modo de resultados y conclusiones, cómo el profesorado pone en acción dichas estrategias como elementos clave para los aprendizajes y la retroalimentación, y de este modo asegurarse que las estrategias de evaluación están alineadas con su práctica en los diferentes contextos.

Palabras clave: competencias digitales, profesorado, evaluación, herramientas digitales, alumnado.

\begin{abstract}
Assessment is a fundamental part of the formative processes of teaching and learning. Teachers and students are called upon to reflect on and assess the deployment of assessment strategies in liquid environments. The aim of this work is to identify digital tools in teachinglearning processes as resources for the acquisition of competences and for educational assessment. To do so, firstly, the concept of assessment is addressed, followed by the techniques and strategies used in educational processes. This is followed by the identification of digital tools and their implementation in the teaching-learning processes, identifying those that, as teachers, we deploy in our educational practices. Finally, we point out, by way of results and conclusions, how teachers put these strategies into action as key elements for learning and feedback, and thus ensure that assessment strategies are aligned with their practice in different contexts.
\end{abstract}

Keywords: digital competences, teachers, assessment, digital tools, students. 


\section{Introducción}

El papel docente en los procesos de enseñanza-aprendizaje es un elemento fundamental para transformar y mejorar las prácticas educativas, y conseguir el éxito en el estudiantado.

Para ello, la evaluación es una pieza clave y es vista como una acción procesual, un conjunto de actividades programadas $\mathrm{y}$, se considera, un proceso sistemático de recogida de datos, incorporado al sistema general de actuación educativa, que permite obtener información válida y fiable para formar juicios de valor acerca de una situación (Cabero-Almenara et al., 2018). Tanto profesorado como alumnado reflexionan y toman decisiones para mejorar sus estrategias de enseñanza y aprendizaje e introducir las mejoras necesarias para que dicho proceso sea verdaderamente formativo (Santos Guerra, 2014). De este modo, la evaluación necesita obtener todos los datos necesarios sobre el proceso investigador por el que se obtienen explicaciones sobre el funcionamiento del proceso de enseñanza-aprendizaje, con el fin de introducir los cambios oportunos para mejorarlo, regular los ritmos y determinar lo positivo del proceso y corregir aquellos puntos mejorables (Mateos, 2000; Vázquez et al., 2011).

Así pues, se trata de una característica de toda actividad humana intencional y requiere objetividad y sistematización, es por ello que exige algunas escalas o criterios que sirvan de referencia. En numerosas ocasiones, la evaluación comienza con la medida, lo que ha supuesto, casualmente, confundir evaluación con medida.

Se puede decir que la evaluación educativa se configura como una acción que debe ser continuada a lo largo de una acción, y requiere de aspectos técnicos, reflexivos y organizativos (Cabero-Almenara et al., 2018). Si esta acción la identificamos, por ejemplo, con un curso escolar, tendríamos: a) evaluación inicial-diagnóstica (comienzo del curso); b) evaluación formativa-procesual (durante el curso); y c) evaluación final-sumativa (final del curso, síntesis integradora). 
Igualmente, tenemos que resaltar que el proceso de evaluación debe abarcar todos los espacios del sistema educativo: los procesos de enseñanza y los de aprendizaje, la práctica docente, los documentos de programación (memorias de verificación de los títulos, programaciones, proyectos docentes...), los diversos elementos del sistema educativo, y los materiales educativos utilizados en la enseñanza.

Una síntesis de los diferentes elementos que componen la evaluación educativa se presenta en la Figura 1.

Figura 1. Dimensiones de la evaluación educativa

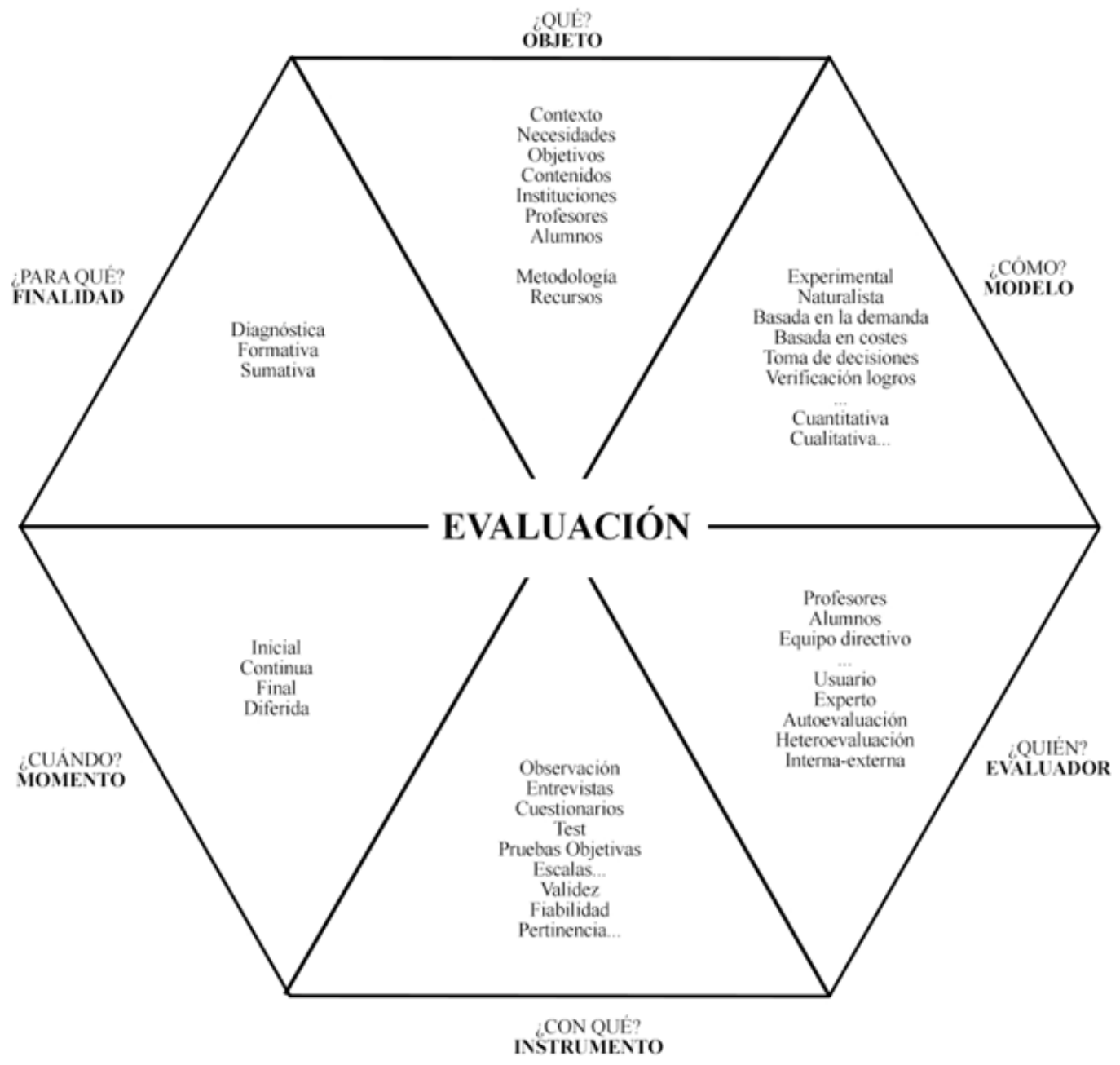

Fuente: Tejada, 1999. 
En cuanto a las estrategias a tener en cuenta a la hora de llevar a cabo el proceso de evaluación, es interesante contemplar los siguientes aspectos:

¿Para qué evaluar? Se debe tener en cuenta que la información que suministra la evaluación debe servir como punto de referencia para tomar medidas de intervención pedagógica. Se evalúa para: mejorar el proceso de aprendizaje e impedir la acumulación de difícultades, modificar el plan de actuación diseñado por el profesor, adoptar medidas de refuerzo educativo o de adaptación curricular, poder intervenir en la resolución de conflictos actitudinales, orientar la actuación tutorial, entre otras. Desde este punto de vista, la evaluación es un proceso que debe llevarse a cabo de forma continua y personalizada. Es decir, integrada en el quehacer diario del proceso de enseñanza-aprendizaje. No puede reducirse a una situación aislada en la que se realizan unas pruebas, sino que es preciso parase a menudo a revisar lo que se está haciendo, dialogar con el alumnado y a reflexionar sobre los ajustes que continuamente se producen. Es personalizada, ya que se tiene en cuenta el desarrollo particular del alumnado, aportándole información sobre lo que realmente ha progresado respecto a sus posibilidades o, en el caso de que se trate de evaluación de materiales o de centros, teniendo en cuenta sus peculiaridades y potencialidades, la facilidad con la que se han comprendido los materiales de enseñanza o la utilidad de las estrategias de enseñanza alcanzada.

¿Qué evaluar? Los criterios de evaluación responden a las capacidades básicas de cada una de las áreas en la etapa y referida a aquellos contenidos específicos que se consideran importantes. Se trata, por lo tanto, de indicadores sobre los que el alumno debe alcanzar un aspecto básico del área que le permita seguir progresando. Estos criterios deben cumplir funciones orientadoras (que nos puede ayudar para la elaboración de proyectos y programaciones al orientar sobre aspectos básicos que el alumno debe alcanzar) y de evaluación, tanto formativa (que ayude a orientar el trabajo futuro) como sumativa (que ayude a la comprobación de los resultados alcanzados y la valoración de su grado de consecución).

¿Cuándo evaluar? En un proceso de evaluación continua del proceso de enseñanzaaprendizaje. Cabe distinguir tres momentos o aspectos distintos y complementarios: 1) 
evaluación inicial (podemos adecuar las intenciones a los conocimientos previos y necesidades de los alumnos, identificando de esta manera el tipo de ayuda más adecuado y adaptado a cada alumno); 2) formativa (podemos ir ajustando la ayuda pedagógica según la información se vaya produciendo; este ajuste sistemático del proceso de enseñanza aprendizaje-aprendizaje requiere que este sea observado sistemáticamente, de tal forma que nos permita detectar los posibles obstáculos que vayan surgiendo, las causas que lo provocan y las correcciones necesarias que debemos introducir) y; 3) sumativa (nos permitirá conocer si el grado de aprendizaje de cada alumno se ha producido o no y, en cualquier caso, cual es el nivel de aprendizaje adquirido).

¿Cómo evaluar? Debemos tener en cuenta que obtener y seleccionar la información para la evaluación exige una reflexión previa sobre la pertinencia de los procedimientos e instrumentos de evaluación, aquellos que mejor se adaptan a las distintas capacidades y a los distintos tipos de contenidos a evaluar. Estos procedimientos deben cumplir algunos criterios como: ser variados, para que nos permitan la contrastar datos; dar información concreta sobre lo que se pretende; en la medida de lo posible, utilizar distintos códigos (verbales, icónicos, gráficos, audiovisuales...); ser aplicables en situaciones habituales de la actividad educativa; evaluar la transferencia de los aprendizajes a contenidos distintos de aquellos en los que se han adquirido, comprobando así su funcionalidad; y ser aplicados, en algunos momentos, tanto por el profesor como por los alumnos (autoevaluación, evaluación entre iguales) de manera individual o grupal.

Las estrategias utilizadas nos deben servir para lograr conocer el desempeño del alumno en un equilibrio entre lo que conoce y lo que es capaz de usar o hacer (Tabla1). 
Tabla 1. Desempeño del alumnado

\begin{tabular}{|l|l|}
\hline Conoce & Usa \\
\hline Recordar & Clasificar \\
\hline Identificar & Comparar \\
\hline Listar & Analizar \\
\hline Definir & Evaluar \\
\hline
\end{tabular}

Las estrategias y técnicas de evaluación que encontramos son diversas, en la Tabla 2 puede observarse una diversidad de ellas y las posibilidades que ofrecen para la evaluación de diferentes aspectos.

Tabla 2. Técnicas, instrumentos y aprendizajes que pueden evaluarse

\begin{tabular}{|c|c|c|c|c|}
\hline \multirow[b]{2}{*}{ Técnicas } & \multirow[b]{2}{*}{ Instrumentos } & \multicolumn{3}{|c|}{ Aprendizajes que pueden evaluarse } \\
\hline & & Conocimientos & Habilidades & $\begin{array}{l}\text { Actitudes/ } \\
\text { valores }\end{array}$ \\
\hline \multirow{5}{*}{ Observación } & Guía de observación & $\sqrt{ }$ & $\checkmark$ & $\sqrt{ }$ \\
\hline & Registro anecdótico & $\sqrt{ }$ & $\checkmark$ & $\checkmark$ \\
\hline & Diario de clase & $\checkmark$ & $\checkmark$ & $\checkmark$ \\
\hline & Diario de trabajo & $\sqrt{ }$ & $\checkmark$ & $\checkmark$ \\
\hline & Escala de actitudes & & & $\checkmark$ \\
\hline \multirow{3}{*}{$\begin{array}{l}\text { Trabajo del } \\
\text { alumnado }\end{array}$} & $\begin{array}{l}\text { Preguntas sobre el } \\
\text { procedimiento }\end{array}$ & $\sqrt{ }$ & $\sqrt{ }$ & \\
\hline & $\begin{array}{lll}\text { Cuadernos } & \text { de } & \text { los } \\
\text { alumnos } & & \\
\end{array}$ & $\sqrt{ }$ & $\checkmark$ & $\sqrt{ }$ \\
\hline & Organizadores gráficos & $\checkmark$ & $\checkmark$ & \\
\hline \multirow{5}{*}{ Desempeño } & Portafolio & $\checkmark$ & $\checkmark$ & \\
\hline & Rúbrica & $\checkmark$ & $\checkmark$ & $\checkmark$ \\
\hline & Lista de cotejo & $\checkmark$ & $\checkmark$ & $\checkmark$ \\
\hline & Proyectos & $\checkmark$ & $\checkmark$ & $\checkmark$ \\
\hline & Mapas mentales & $\checkmark$ & $\sqrt{ }$ & \\
\hline
\end{tabular}




\begin{tabular}{|c|c|c|c|c|}
\hline \multirow{2}{*}{$\begin{array}{l}\text { Pruebas } \\
\text { específicas }\end{array}$} & $\begin{array}{l}\text { Tipos textuales: Debate } \\
\text { y Ensayo }\end{array}$ & $\checkmark$ & $\sqrt{ }$ & $\checkmark$ \\
\hline & $\begin{array}{l}\text { Tipos orales y escritos: } \\
\text { Pruebas } \\
\text { escritas }\end{array}$ & $\checkmark$ & $\checkmark$ & \\
\hline
\end{tabular}

\section{La evaluación de competencias}

Las competencias digitales docentes (CDD) es multidimensional (Flores \& Roig, 2019), así pues, tal y como señala Durán (2019, p. 27) se trata de "conjunto de conocimientos, capacidades y actitudes necesarios para que un docente haga un uso efectivo de las TIC desde sus distintas vertientes (tecnológica, informacional, multimedia, comunicativa, colaborativa y ética), asumiendo criterios pedagógico-didácticos para una integración efectiva de éstas en su experiencia docente."

En cuanto a la evaluación de competencias Castro (2010) la define como "un procedimiento en el que se requiere que el estudiante complete tareas o procesos en los que se demuestre su habilidad para aplicar conocimiento y destrezas o aplicar conocimientos en situaciones simuladas similares a la vida real” (p. 118). En este sentido, abogamos por la evaluación de competencias no centradas en el aprendizaje; sino más bien, para el aprendizaje.

\section{Herramientas digitales para evaluar}

Teniendo en cuenta el análisis de las estrategias de evaluación, el objetivo de este trabajo es identificar herramientas digitales en los procesos de enseñanza-aprendizaje como recursos para la adquisición de competencias y para evaluación educativa. La evaluación de los aprendizajes puede apoyarse en la posibilidad que le ofrecen diferentes herramientas digitales. Por ejemplo, para la realización de encuestas y exámenes en línea. Si el docente recurre a los dispositivos digitales que suelen poseer sus estudiantes, puede hacer actividades gamificación para analizar lo aprendido en la clase o el análisis de los conceptos por aprender. 
Otra forma de utilizar las herramientas digitales es crear un entorno digital, como un blog, para que los estudiantes puedan publicar sus ideas y muestren ejemplos de su trabajo. De esta forma, se puede combinar la evaluación y autorreflexión por parte del estudiante. Esta forma de utilizar las tecnologías digitales para evaluación permitirá también aplicar otras estrategias, como la evaluación entre iguales (Panadero et al., 2016).

Sin embargo, al igual que ocurrió con la enseñanza asistida por ordenador, la evaluación informatizada ha producido una serie de términos y siglas para referirse a ella. Podemos encontrar estudios (Stephens \& Mascia, 1995; Brown et al., 1997; McKenna \& Bull, 1999) en los que se distingue entre:

. Computer-Assisted Assessment (CAA) se refiere al empleo de los ordenadores en algún momento del proceso de evaluación en: la distribución, análisis y puntuación de los trabajos y exámenes; el análisis de los datos a través de software que nos permite procesar de manera rápida los datos; el almacenamiento, análisis e informe en la ejecución y; el análisis y transferencia de la información de la evaluación a través de la red.

. Computer-Based Assesssment (CBA) se refiere a la completa automatización del proceso evaluativo. Las preguntas o las tareas se presentan al estudiante a través de un ordenador, este responde por medio del teclado, y las calificaciones se le entregan electrónicamente.

Igualmente, con el uso cada vez más frecuente de las redes, se está produciendo una variación en la nomenclatura anteriormente comentada, empezándose a extender el empleo de los términos Web-Assisted Assessment y Web-Based Assissment.

El uso de tecnologías en el proceso de evaluación permite incrementar la eficacia de la misma, aminorar el tiempo para corregir y aumentar la fiabilidad. Además de aplicar nuevas estrategias evaluativas.

En la Tabla 3 se encuentran herramientas fáciles de usar por parte del docente, clasificadas por Educación 3.0 (2017). Éstas permiten calificar al estudiantado a través del ordenador, 
tablets o incluso smartphones con juegos y test personalizados que permitirán repasar desde un tema hasta una asignatura completa.

Tabla 3. Herramientas que facilitan la evaluación del proceso enseñanza-aprendizaje

\begin{tabular}{|c|c|c|}
\hline Nombre & Descripción & Imagen \\
\hline Socrative & $\begin{array}{l}\text { Esta herramienta permite realizar concursos de preguntas y respuestas de } \\
\text { forma trivial para que el alumnado participe en ellos. Teniendo en cuenta } \\
\text { que necesita de un registro previo, toda información puede ser seguida por } \\
\text { el profesorado. }\end{array}$ & \\
\hline Plickers & $\begin{array}{l}\text { Plickers es una plataforma para evaluar a al estudiantado de múltiples } \\
\text { modos. Por ejemplo, podremos hacerles una pregunta y ellos responderán } \\
\text { levantando una tarjeta con un código que leeremos a través de la cámara } \\
\text { de un smartphone o tablet, con una app específica que corregirá las } \\
\text { respuestas de cada alumno dándonos toda la información al instante. } \\
\text { También se pueden crear clases para llevar un registro de su evolución o } \\
\text { lanzar preguntas con diferentes tipos de respuestas. }\end{array}$ & Pli \\
\hline Kahoot & $\begin{array}{l}\text { Plataforma en línea que mezcla juego con entretenimiento y diversión. } \\
\text { Kahoot nos permite crear 'quizs' on line en los que el estudiantado puede } \\
\text { participar, ya sea por separado o en equipo. Se crea un conjunto de } \\
\text { preguntas, junto con sus respectivas respuestas, que no siempre serán } \\
\text { verdaderas. Al final del juego, hay un ranking con los mejores jugadores } \\
\text { de cada partida, lo que nos puede servir para evaluar el conjunto. }\end{array}$ & Kahoot! \\
\hline EDPuzzle & $\begin{array}{l}\text { Permite crear vídeos y añadir texto o comentarios a ellos, también } \\
\text { podremos evaluar al alumnado de una forma sencilla. Por ejemplo, } \\
\text { añadiendo ciertas preguntas a lo largo del vídeo, la plataforma registra las } \\
\text { respuestas de cada alumno para que luego nosotros podamos determinar } \\
\text { su nivel de comprensión respecto de la materia. }\end{array}$ & 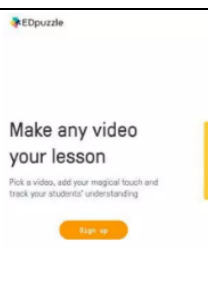 \\
\hline
\end{tabular}




\begin{tabular}{|c|c|c|}
\hline Google Forms & $\begin{array}{l}\text { Permite crear formularios on line de forma sencilla para realizar una } \\
\text { evaluación de contenidos. Necesitaremos, eso sí, crear un formulario } \\
\text { acorde al objetivo que queramos plantear, pero una vez creado, tendremos } \\
\text { toda la información en una hoja de cálculo que nos permitirá jugar con } \\
\text { toda esta información, sacar estadísticas o corregirlos de forma } \\
\text { prácticamente automática. }\end{array}$ & \\
\hline Edmodo & $\begin{array}{l}\text { Permite crear tests muy personalizables para evaluar el nivel del alumnado } \\
\text { sobre un cierto tema-materia. Pueden ser preguntas con diferentes tipos de } \\
\text { respuestas, todo ello automatizado y con una guía en la que nos cuentan } \\
\text { cómo hacerlo. }\end{array}$ & 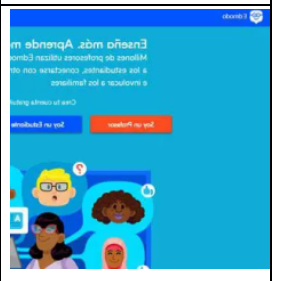 \\
\hline Triv & $\begin{array}{l}\text { Permite que tanto estudiantado como profesorado elaboren sus propias } \\
\text { preguntas y respuestas. Esto también puede utilizarse con una finalidad } \\
\text { evaluadora, ya que se podrá crear un trivial específico para el alumnado, } \\
\text { adecuado a la lección y asignatura que quieras. }\end{array}$ & 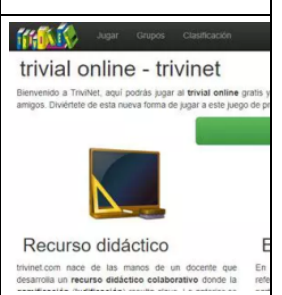 \\
\hline Nearpod & $\begin{array}{l}\text { Herramienta con múltiples posibilidades. Una de ellas permite evaluar al } \\
\text { estudiantado a través de un dispositivo móvil, tableta u ordenador. El } \\
\text { docente puede crear una lección personalizada a todo lo que desee para, a } \\
\text { partir de ella, elaborar una serie de pruebas y preguntas con las que poder } \\
\text { saber el conocimiento adquirido por el alumnado. }\end{array}$ & $\begin{array}{l}\text { pls for the most } \\
\text { job in the } w \\
\text { or reachess }\end{array}$ \\
\hline Formative & $\begin{array}{l}\text { Es una app que permite enviar preguntas, lecciones y todo tipo de material } \\
\text { al estudiantado para que, a través de sus tabletas, puedan resolverlas al } \\
\text { instante. El profesorado puede ver, en tiempo real, las respuestas de cada, } \\
\text { lo que le permitirá evaluarles inmediatamente. }\end{array}$ & itudent Growth Mad \\
\hline Flipquiz & $\begin{array}{l}\text { El profesorado puede crear un quiz y enviárselo al alumnado, que jugará } \\
\text { individualmente o en grupo, a modo de concurso. Bien configurados los } \\
\text { equipos, puede ser un momento muy divertido y didáctico dentro de la } \\
\text { clase. }\end{array}$ & 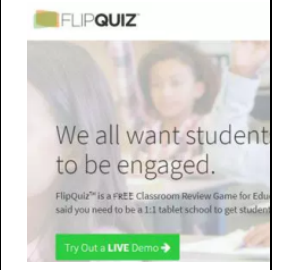 \\
\hline
\end{tabular}




\begin{tabular}{|c|c|c|}
\hline Mentimeter & $\begin{array}{l}\text { Esta plataforma permite la creación de encuestas mediante la inclusión, } \\
\text { primero, de la pregunta y, a continuación, de las distintas respuestas entre } \\
\text { las que el alumnado debe elegir la correcta. Proporciona un panel de } \\
\text { gestión que hace posible cualquier modificación y desde el que también se } \\
\text { pueden comprobar los resultados finales. Se puede acceder a él a través del } \\
\text { ordenador o desde una tableta o Smartphone. }\end{array}$ & \\
\hline Quizalize & $\begin{array}{l}\text { Creación de exámenes on line desde cero, o tomando alguno creado por } \\
\text { terceros, con las preguntas que el alumnado debe responder (en el aula o } \\
\text { en casa). El docente puede seguir el proceso en tiempo real a través del } \\
\text { Panel del Profesor, entregando puntos al estudiantado que responde } \\
\text { correctamente las preguntas en primer lugar. La puntuación total se entrega } \\
\text { cuando todos los participantes hayan concluido. }\end{array}$ & \\
\hline Edulastic & $\begin{array}{l}30 \text { tipos de preguntas interactivas y multimedia, el docente puede crear } \\
\text { cuestionarios on line en los que incluir contenido adicional tanto fijos } \\
\text { (texto, imágenes o gráficos) como en movimiento (vídeo). También } \\
\text { permite incrustar enlaces web. Cuando el estudiantado termina de } \\
\text { responder las preguntas, el profesorado recibe los resultados de forma } \\
\text { inmediata junto a una comparativa con los estándares establecidos. }\end{array}$ & th \\
\hline Quizzizz & $\begin{array}{l}\text { Plataforma gratuita de gamificación habilita al profesorado la creación de } \\
\text { test on line, así como su envío al alumnado mediante su navegador web. } \\
\text { Para su edición, cuenta con dos modalidades, en vivo y en casa, y puede } \\
\text { personalizarse en base a varios criterios como el número de respuestas o el } \\
\text { tiempo para responderlas. Tras su cumplimentación, el profesor recibe un } \\
\text { informe con los resultados de cada alumno y de la clase, con los que } \\
\text { dictaminar los puntos a mejorar. }\end{array}$ & \\
\hline Classkick & $\begin{array}{l}\text { Desde su página web, el profesorado puede crear una tarea personalizada } \\
\text { para cada alumno, o genérica para toda la clase, y seguir su desarrollo a } \\
\text { través de su dispositivo. De este modo, puede evaluar la forma de } \\
\text { completar cada ejercicio por parte de sus alumnos o ver si las respuestas } \\
\text { son las correctas. Al final del proceso, recibe un documento con todos los } \\
\text { resultados. Cuenta con una versión móvil, apta sólo para terminales con } \\
\text { iOS. }\end{array}$ & $\mathbf{c l a s s k i c k}_{\mathbf{C}}$ \\
\hline
\end{tabular}

Fuente: Adaptado de Educación 3.0 
También se potencia que el estudiantado colabore en la realización de las encuestas como tarea colaborativa de clase aportando ítems, y que la versión final sea elaborada por un grupo de la clase.

Es importante reflexionar sobre la utilidad de la información que se está recogiendo mediante las encuestas. En caso necesario, se pueden utilizar otras propuestas digitales como un blog o portafolios digitales donde el alumnado publica ejemplos de sus trabajos. Esta estrategia permitirá combinar la evaluación con la autoevaluación y la evaluación por pares.

Para aumentar la variedad y la adecuación el docente, puede experimentar diferentes formatos, como las rúbricas de evaluación, retroalimentación simbólica (estrellas o "me gusta", retroalimentación en video o audio, entre otras).

Por último, consideramos que el docente reflexione y analice en torno a las posibilidades que le ofrecen las herramientas del campus virtual de su universidad para obtener información estructurada de sus estudiantes.

\section{Discusión y conclusiones}

Del presente discurso, se desprende que la evaluación del alumnado se realiza a partir de una gran variedad de herramientas digitales que le permite al docente no solo la presentación de una calificación final, sino el monitoreo de todo el proceso de enseñanza-aprendizaje, proporcionándole una evaluación que debe ser:

. Explicita y clara: el modo y los criterios de evaluación deben ser claros, públicos y conocidos. . Válida: mide lo que ha marcado medir.

- Consistente: se obtiene con ella, de forma constante, información sobre el cambio que queremos medir.

. Flexible: emplea métodos diversos para necesidades diversas.

. Justa: los mismos criterios para todos los evaluados. 
- Coherente: que no esté disociada de la metodología del curso ni del medio que se ha empleado en la acción docente.

. Constructiva: Aporte elementos a la construcción que hace el estudiante de su conocimiento.

. Propia: Posibilidad de autoevaluación por parte del estudiante.

. Formativa: La evaluación forma parte del proceso de aprendizaje.

La evaluación debe ser entendida como un proceso (tanto para el profesor como para los estudiantes) concebido como una oportunidad formativa cuya finalidad prioritaria sea que los estudiantes adquieran conocimientos y desarrollen y consoliden competencias, tal y como manifestaban Mateos (2000), Blázquez et al. (2017) y Cabero-Almenara et al. (2018). Una evaluación centrada en ayudar a aprender (Padilla y Gil, 2008), una evaluación formativa.

En esta línea, las tecnologías pueden brindar al docente grandes oportunidades a la hora de enfrentarse a la evaluación formativa, ayudándole a proporcionar la información necesaria (retroalimentación) para ajustar sobre la marcha dichos procesos de forma que los estudiantes logren alcanzar los objetivos propuestos (Morales Vallejo, 2008).

También puede hacer que los estudiantes colaboren en la realización de las encuestas como tarea colaborativa de clase aportando ítems, y que la versión final sea elaborada por un grupo de la clase.

Es importante reflexionar sobre la utilidad de la información que se está recogiendo mediante las encuestas. En caso necesario, se pueden utilizar otras propuestas digitales como un blog o portafolios digitales donde los alumnos publiquen ejemplos de sus trabajos. Esta estrategia le permitirá combinar la evaluación con la autoevaluación y la evaluación por pares.

Para aumentar la variedad y la adecuación el docente puede experimentar diferentes formatos, como las rúbricas de evaluación, retroalimentación simbólica (estrellas o "me gusta", retroalimentación en video o audio). 
También es importante en este nivel que el docente comience a analizar las posibilidades que le ofrecen las herramientas del campus virtual de su universidad para obtener información estructurada de sus estudiantes.

En este nivel, el docente debe establecer estrategias para conocer si su alumnado aprecia las estrategias utilizadas para dar retroalimentación y asegurarse que las estrategias de evaluación están alineadas con su práctica.

Es importante también ir probando y utilizando las diversas herramientas que el campus virtual de la institución educativa pone a disposición de sus docentes.

Puede ser también de utilidad la utilización de estrategias de evaluación cualitativa mediante herramientas digitales. En este sentido, Blázquez et al. (2017) exponen las siguientes posibilidades:

- Entrevistas y grupos de debate on-line. Para estas entrevistas, puede utilizar diferentes herramientas digitales como: Zoom, Skype, Adobe Connect... Estas herramientas permiten grabar y analizar posteriormente con tranquilidad la entrevista realizada.

. Cuestionarios abiertos digitales. Para ello, se pueden utilizar diferentes herramientas como SurveyMonkey, Google Forms, Eval\&go...

. Portafolios electrónicos. Para ello, puede utilizar herramientas como Google Drive, Dropbox, Eduporfolio...

. Infografía digital y mapas mentales. Para ello, puede utilizar diferentes herramientas como Genial.ly, Infogram, Easel.ly, Canva, Popplet, Crately, ...

- Narraciones digitales (digital storytelling). Para ello, puede apoyarse en programas como Movie Maker, iMovie...

. Diario de campo digital con edublog. Para ello, puede utilizar herramientas tradicionales para la creación de blog como Edublog y Blogger. 
De esta manera y, de acuerdo con Lipsman (2009), el uso de herramientas tecnológicas puede ayudar a extender y ampliar los procesos cognitivos de los estudiantes que contribuyen a la resolución de problemas complejos y les brindan oportunidades para colaborar e investigar. Finalmente, señalar de acuerdo con Biggs (2006), que podemos poner en acción dos dimensiones de evaluaciones:

. Evaluaciones descontextualizadas, como un examen escrito o un trabajo de trimestre, que son aptas para evaluar el conocimiento declarativo.

- Evaluaciones contextualizadas, como unas prácticas, la resolución de problemas o el diagnóstico de un estudio de casos, que son adecuadas para evaluar el conocimiento funcional.

\section{Referencias}

Biggs, M. J. G. (2006). Comparison of student perceptions of classroom instruction: Traditional, hybrid, and distance education. Turkish Online Journal of Distance Education, 7(2), 46-51.

Blazquez, F, Alonso, L. \& Yuste, R. (2017). Evaluación en la era digital. Síntesis.

Brown, G., Bull, J. \& Pendlebury, M. (1997). Assessing Student Learning in Higher Education. Routledge.

Cabero Almenara, J., Llorente Cejudo, C. \& Morales Lozano, J. A. (2018). Evaluación del desempeño docente en la formación virtual: ideas para la configuración de un modelo. RIED. Revista Iberoamericana De Educación a Distancia, 21(1), 261-279. https://doi.org/10.5944/ried.21.1.17206

Castro, M. (2010). ¿Qué sabemos de la medida de lascompetencias? Características y problemas psicométricos en la evaluación de competencias. Bordón, 63(1), 109-123. 
Durán, M. (2019). Competencia Digital del Profesorado Universitario: diseño y validación de un instrumento para la certificación. Universidad de Murcia. [Tesis doctoral inédita]. Educación 3.0 (2017). 15 herramientas para evaluar a los estudiantes. https://www.educaciontrespuntocero.com/recursos/herramientas-evaluar-estudiantes

Flores, C. \& Roig, R. (2016). Diseño y validación de una escala de autoevaluación de competencias digitales para estudiantes de pedagogía. Píxel-Bit. Revista de Medios y Educación, 12(48), 209-224. https://doi.org/10.12795/pixelbit.2016.i48.14

Lipsman, N. (2009). Personal identity, enhancement and neurosurgery: a qualitative study in applied neuroethics. Bioethics, 23(6), 375-383.

Mateos, J. (2000). La evaluación educativa. Su práctica y otras metáforas. ICE-UB-Octaedro.

McKenna, C. \& Bull, J. (1999). Frecuently asked question about CAA. http://www.caacentre.ac.uk

Padilla, M.T., y Gil, J. (2008). La evaluación orientada al aprendizaje en la Educación Superior: condiciones y estrategias para su aplicación en la docencia universitaria. Revista Española de Pedagogía, 66(241), 467-486.

Panadero, E., Jonsson, A. \& Strijbos, J. W. (2016). Scaffolding self-regulated learning through self-assessment and peer assessment: Guidelines for classroom implementation. In D. Laveault \& L. Allal (Eds.). Assessment for Learning: Meeting the challenge of implementation (pp. 311-326). Springer International Publishing.

Santos Guerra, M. A. (2014). La Evaluación como Aprendizaje. Cuando la flecha impacta en la diana. Narcea. 
Stephens, D. \& Mascia, J. (1995). Results of a survey into the use of computer assisted assessment in institutions of Higher Education in the UK. Loughborough: Flexible Learning Initiative.

Tejada, J. (1999). La evaluación: su conceptualización. Síntesis.

Vázquez, P., Ortega, J. L., Sánchez, F. \& Ibáñez, G. (2011). Competencias básicas: desarrollo y evaluación en educación secundaria. Octaedro. 